EGU2020-10227, updated on 22 Dec 2021

https://doi.org/10.5194/egusphere-egu2020-10227

EGU General Assembly 2020

(c) Author(s) 2021. This work is distributed under

the Creative Commons Attribution 4.0 License.

\title{
Towards an ontology based conceptual model, establishing maximum interoperability for interactive and distributed processing of geoscientific information
}

\author{
Rainer Haener ${ }^{1}$, Henning Lorenz ${ }^{2}$, Sylvain Grellet ${ }^{3}$, Marc Urvois ${ }^{3}$, and Eberhard Kunz ${ }^{4}$ \\ ${ }^{1}$ Helmholtz Centre Potsdam GFZ German Research Centre for Geosciences, ID 2, Potsdam, Germany \\ ${ }^{2}$ Uppsala University, Department of Earth Sciences, Uppsala, Sweden \\ ${ }^{3}$ The French Geological Survey, Orleans, France \\ ${ }^{4}$ Federal Waterways Engineering and Research Institute, Karlsruhe, Germany
}

This study presents an approach on how to establish Conceptual Interoperability for autonomous, multidisciplinary systems participating in Research Infrastructures, Early Warning, or Risk Management Systems. Although promising implementations already exist, true interoperability is far from being achieved. Therefore, reference architectures and principles of Systems-of-Systems are adapted for a fully specified, yet implementation-independent Conceptual Model, establishing interoperability to the highest possible degree. The approach utilises use cases and requirements from geological information processing and modelling within the European Plate Observing System (EPOS).

Conceptual Interoperability can be accomplished by enabling Service Composability. Unlike integration, composability allows interactive data processing and beyond, evolving systems that enable interpretation and evaluation by any potential participant. Integrating data from different domains often leads to monolithic services that are implemented only for a specific purpose (Stovepipe System). Consequently, composability is essential for collaborative information processing, especially in modern interactive computing and exploration environments. A major design principle for achieving composability is Dependency Injection, allowing flexible combinations (Loose Coupling) of services that implement common, standardised interfaces (abstractions). Another decisive factor for establishing interoperability are Metamodels of data models that specify data and semantics regardless of their domain, based on a common, reusable approach. Thus, data from different domains can be represented by one common encoding that e.g. abstracts landslides (geophysical models) or buildings (urban planning) based on their geometry. An indispensable part of a Conceptual Model is detailed semantics, which not only requires terms from Domain-Controlled Vocabularies, but also ontologies providing qualified statements about the relationship between data and associated concepts. This is of major importance for evolutionary systems that are able to comprehend and react to state changes. Maximum interoperability also requires strict modularisation for a clear separation of semantics, metadata and the data itself. 
Conceptual models for geological information that are governed by the described principles and their implementations are still far away. Moreover, a route to achieve such models is not straightforward. They span a multitude of communities and are far too complex for conventional implementation in project form. A first step could be applying modern design principles to new developments in the various scientific communities and join the results under a common stewardship like the Open Geospatial Consortium (OGC). Recently, a Metamodel has been developed within the OGC's Borehole Interoperability Experiment (BoreholelE); initiated and led by the French Geological Survey (BRGM). It combines the ISO standard (19148:2012 linear referencing) for localisation along borehole paths with the adaption of different encodings of borehole logs based on well-established OGC standards. Further developments aim at correlating borehole logs, geological or geotechnical surveys, and geoscientific models. Since results of surveys are often only available as non-schematised interpretations in text form, interoperability requires formal classifications, which can be derived from machine learning methods applied to the interpretations. As part of a Conceptual Model, such classifications can be used for an automated exchange of standard-conform borehole logs or to support the generation of expert opinions on soil investigations. 